\section{Mínima intervención en Odontología: ¿una moda emergente en tiempos de pandemia?}

\section{Minimal Intervention in Dentistry: An emerging trend during Pandemic?}

\section{Señor Editor,}

Durante los últimos meses, la Odontología es probablemente una de las áreas médicas que más ha sido impactada por la pandemia mundial por la COVID-19 (1). Diversas organizaciones recomendaron suspender toda actividad clínica debido al riesgo de infección por transmisión del SARS-CoV-2. De esa forma, la atención odontológica fue limitada a la resolución de emergencias y urgencias dentales impostergables. En ese minuto, miles de odontólogos en el mundo se vieron forzados a adecuar su actuar profesional acorde con las regulaciones ${ }^{1} \mathrm{y}$ comenzaron a explorar nuevas posibilidades de desarrollar la profesión bajo el contexto de pandemia ${ }^{2}$. Así, la mínima intervención en odontología $(\mathrm{MI})^{3}$ comenzó a ocupar un rol protagónico.

La preservación de la dentición se relaciona con la calidad de vida ${ }^{4}$, influyendo en distintas esferas de la vida como bienestar físico, psicológico, social, e incluso con la memoria y la capacidad cognitiva futura. Dada la importancia de preservar la dentición y que los dientes están conformados por tejidos no regenerables, los esfuerzos de la profesión deben orientarse a evitar y no a reparar el daño ${ }^{5}$. Además, debido a que las restauraciones tienen una vida media limitada, y un alto costo, existe acuerdo y evidencia científica sobre los beneficios y ventajas de implementar estrategias con una filosofía de $\mathrm{MI}^{3}$. A pesar de que parece evidente que la enfermedad de caries debe ser manejada controlando sus agentes causales; la dieta y el biofilm dental, las restauraciones han sido considerado el tratamiento estándar para caries dental. La reciente actualización de la definición de caries deberá impactar su manejo ${ }^{6}$. La caries dental pasó de ser considerada una enfermedad infecciosa, a ser entendida como enfermedad crónica no transmisible, con un fuerte componente conductual ${ }^{6}$. Debido a este nuevo entendimiento, el foco de la intervención se basa en el cambio conductual, preferentemente relacionado con decisiones alimentarias (dieta). La dieta, factor común para muchas enfermedades crónicas no transmisibles, conlleva beneficios que van mucho más allá de la cavidad oral. Así, las maniobras de $\mathrm{MI}^{3}$ destinadas al control de la enfermedad, bajo el nuevo foco del manejo de la enfermedad de caries, incluyen (a) Terapias no invasivas (educación en salud, instrucción de higiene oral, asesoramiento en dieta, uso racional de fluoruros, e intervenciones para el cambio de conducta y el aumento de la autoeficacia), (b) micro invasivas (sellantes e infiltrantes), y (c) mínimamente invasivas (técnica atraumática, uso de flúor diamino de plata para manejo de lesiones cavitadas, entre otras. Además, el acceso ubicuo a tecnología ha permitido realizar atenciones de forma remota (TeleOdontología), preferentemente desde la prevención. Dado que la evidencia es concluyente sobre las ventajas de la $\mathrm{MI}^{2,3,5}$, este tipo de terapias debiese ser lo habitual en la práctica clínica. La situación impuesta por la pandemia debiese reforzar y priorizar esta filosofía, toda vez que estas técnicas no generan aerosoles. Sin embargo, lo que actualmente podría considerarse una "moda circunstancial" debiese ser mantenido post-pandemia.

En otra arista del problema, el número de actividades de educación continua, y actualización en temas relacionados a MI han aumentado exponencialmente. La masificación de videoconferencias de carácter gratuito, publicaciones científicas ${ }^{2}$, y difusión en redes sociales, ha permitido que un gran número de profesionales haya podido conocer las nuevas tendencias de MI para el manejo de la enfermedad. Es tal el interés en esta área, que sociedades profesionales con un enfoque típicamente curativo y rehabilitador (invasivo) también están incorporando MI en sus encuentros. Sin embargo, hasta que los odontólogos de Chile y el mundo no asuman su rol promotor de salud para empoderar a los pacientes en su autocuidado, se continuará limitando el daño una vez que este se ha producido, impidiendo el logro del objetivo de mantener la integridad de los tejidos dentales a través del curso de vida. El MINSAL ha recogido la evidencia y ha incorporado un enfoque de MI y preventivo (aun cuando enfocado en la infancia) en sus programas. De hecho, las metas para medir el impacto de atención están siendo reformulados con foco en la prevención más que en la reparación del daño. Cambios de esa envergadura potenciarán el uso de modalidades de MI transversalmente en el servicio público y privado, mejorando la salud de las personas, y contribuyendo a reducir las inequidades en salud.

La pandemia ha sido un fenómeno sin precedentes que ha obligado a la reflexión sobre la profesión, pero también sobre la vida personal, posiblemente modificando nuestras prioridades. Es así como el foco centrado en la estética por parte de pacientes y profesionales podría dar un giro hacia valorar el mantener la salud y desistir de intervenciones invasivas que provocan desgastes de tejido sano (irrecuperable) solo con fines estéticos. Probablemente pacientes que han sufrido episodios dolorosos, con la dificultad de acceso a la atención producto de la pandemia, podrían estar decididos a modificar sus conductas para cuidar y recuperar su salud. Si se considera el costo asociado al manejo de las secuelas de la enfermedad, es claro que el foco debiese estar en la prevención. Hasta ahora, la práctica profesional ha sido basada y medida por la productividad de actividades invasivas (ej. número de restauraciones). Esto trae como consecuencia que 
los pacientes normalicen y consideren que el trabajo del odontólogo se restringe a "tapar" caries (ej.: "si el dentista no usó la "maquinita", el dentista no hizo nada"). La contingencia nos ha llamado a reflexionar como queremos ejercer nuestro rol profesional. Positivamente esta pandemia también nos ha enseñado que los Odontólogos podemos ejercer nuestra profesión a distancia (teleodontología), y sin el uso del temido instrumental rotatorio (turbina).

Las nuevas generaciones de estudiantes de odontología son un campo fértil para incorporar esta filosofía de MI. De hecho, en el reciente lanzamiento de los contenidos mínimos del currículum de Odontología en nuestro país, se llamó a formar odontólogos con un enfoque menos inclinado al desarrollo de destrezas manuales para la ejecución de acciones invasivas. Es evidente que las nuevas generaciones de odontólogos tienen un enfoque más altruista y filantrópico. En lo personal, anhelo que la $\mathrm{MI}^{3}$ no sea solo una moda en tiempos de pandemia, sino que permanezca y aumente su uso, ya sea de manera remota (teleodontología) o presencial. De esta forma estaremos preservando y recuperando salud, manteniendo un tejido irremplazable y contribuyendo a la calidad de vida de las personas.

Constanza E. Fernández ${ }^{1}$

${ }^{1}$ Cirujano Dentista, Doctora en Odontología-Cariología. Académica Investigadora, Dep. Rehabilitación

Buco-Maxilofacial. Escuela de Odontología, Facultad de Ciencias de la Salud, Universidad de Talca, Chile.

\section{Referencias}

1. Coulthard P. Dentistry and coronavirus (COVID-19) - moral decision-making. Br Dent J 2020; 228 (7): 503-5.

2. León S, Giacaman RA. COVID-19 and Inequities in Oral Health Care for Older People: An Opportunity for Emerging Paradigms. JDR Clin Trans Res. 2020:2380084420934742.

3. Innes NPT, Chu CH, Fontana M, Lo ECM, Thomson WM, Uribe S, et al. A Century of Change towards Prevention and Minimal Intervention in Cariology. J Dent Res 2019; 98 (6): 611-7.

4. Schierz O, Baba K, Fueki K. Functional oral health-related quality of life impact: A systematic review in populations with tooth loss. J Oral Rehabil. 2020. Online ahead of print.

5. Innes NPT, Schwendicke F. Restorative Thresholds for Carious Lesions: Systematic Review and Meta-analysis. J Dent Res 2017; 96 (5): 501-8.

6. Fernández CE, Chanin M, Appice GM, Culver AM, Stein A. Conceptualization of dental caries by dental students is related to their preventive oral care routine. J Dent Educ 2020. Online ahead of print.

Correspondencia a:

Dr. Constanza E. Fernández, DDS, PhD

Escuela de Odontología, Universidad de Talca,

Av Lircay S/N, Talca, Chile

cfernandez.go@gmail.com,cofernandez@utalca.cl 